\title{
Action video game experience reduces the cost of switching tasks
}

\author{
Matthew S. Cain • Ayelet N. Landau • \\ Arthur P. Shimamura
}

Published online: 14 March 2012

(C) Psychonomic Society, Inc. 2012

\begin{abstract}
Video game expertise has been shown to have beneficial effects for visual attention processes, but the effects of action video game playing on executive functions, such as task switching and filtering out distracting information, are less well understood. In the main experiment presented here, video game players (VGPs) and nonplayers (nVGPs) switched between two tasks of unequal familiarity: a familiar task of responding in the direction indicated by an arrow, and a novel task of responding in the opposite direction. nVGPs had large response time costs for switching from the novel task to the familiar task, and small costs for switching from the familiar task to the novel task, replicating prior findings. However, as compared to the nVGPs, VGPs were more facile in switching between tasks, producing overall smaller and more symmetric switching costs, suggesting that experience with action video games produces improvements in executive functioning. In contrast, VGPs and nVGPs did not differ in filtering out the irrelevant flanking stimuli or in remembering details of aurally presented stories. The lack of global differences between the groups suggests that the improved task-switching performance seen in VGPs was not due to differences in global factors, such as VGPs being more motivated than nVGPs.
\end{abstract}

M. S. Cain · A. N. Landau • A. P. Shimamura

Department of Psychology, University of California,

Berkeley, CA, USA

\author{
A. N. Landau \\ Ernst Strüngmann Institute (ESI) in \\ Cooperation with Max Planck Society, \\ Frankfurt, Germany \\ M. S. Cain $(\square)$ \\ Center for Cognitive Neuroscience, Duke University, \\ B203 Levine Science Research Center, Box 90999, \\ Durham, NC 27708, USA \\ e-mail: matthew.s.cain@duke.edu
}

Keywords Attention - Executive control

Video games are a widespread pastime, but research has demonstrated that, beyond entertainment value, video game play can provide a variety of potential improvements in visual attention abilities (Dye, Green, \& Bavelier, 2009a; Green \& Bavelier, 2003; but see Boot, Blakely, \& Simons, 2011; Boot, Kramer, Simons, Fabiani, \& Gratton, 2008; Irons, Remington, $\&$ McLean, 2011; and Murphy \& Spencer, 2009, for qualifications of these findings), sensory integration abilities (Donohue, Woldorff, \& Mitroff, 2010), and skills such as mental rotation (Okagaki \& Frensch, 1994) and smooth pursuit (Tsoi, Koopman, \& Wilmer, 2011). While other studies have examined higher-level differences, such as attentional control (Chisholm, Hickey, Theeuwes, \& Kingstone, 2010) and change detection strategy (Clark, Fleck, \& Mitroff, 2011), the effects of video game experience on most higher-level cognitive abilities are not well understood. Here, we compared expert and novice video game players (VGPs and nVGPs) on three key cognitive abilities: task switching, distractor filtering, and logical memory.

The ability to flexibly switch between tasks has been studied extensively (e.g., Allport, Styles, \& Hsieh, 1994; Jersild, 1927; Spector \& Biederman, 1976; Wylie \& Allport, 2000). Switching tasks incurs costs in response times (RTs) and/or error rates as compared to repeating a task. Costs can be reduced by using predictable switches or precues identifying the upcoming task, but some residual costs remain (see Monsell, 2003, for a review). When comparing tasks of unequal difficulty, task switch costs are larger when switching from a difficult task to an easier task than vice versa (e.g., Monsell, Yeung, \& Azuma, 2000). Using tasks of unequal difficulty allows us to answer two distinct questions: Do overall differences in task-switching costs exist between VGPs and nVGPs, and do VGPs and nVGPs differ in the 
symmetry of their task-switching costs? An overall difference would indicate that action video game experience likely transfers to real-life situations that require frequent task switches, such as driving, but the finding could also be due to other factors, such as differences in processing speed (e.g., Dye, Green, \& Bavelier, 2009b). Importantly, a difference in task switch asymmetry would suggest that differences between VGPs and nVGPs are specifically due to changes to executive control processes, rather than to generally increased speed of processing.

Several previous studies have argued that VGPs outperform nVGPs at task switching, but the findings have been inconsistent. Andrews and Murphy (2006) found reduced switching costs for VGPs, but only at short intertrial intervals (ITIs). Two other studies found reduced switching costs for VGPs using task precues (Karle, Watter, \& Shedden, 2010) and predictable task orders (Colzato, van Leeuwen, van den Wildenberg, \& Hommel, 2010). In the present experiment, we investigated task-switching performance in VGPs and nVGPs with long ITIs, no precues, and an unpredictable task sequence.

Alongside frequent task shifts, many video games include considerable irrelevant visual information, and experience in such environments might lead to improved filtering of distractions. However, a previous flanker task study found no differences between VGPs and nVGPs with low perceptual loads, and reduced distractor filtering for VGPs at high perceptual loads (Green \& Bavelier, 2003). In a recent set of flanker experiments, Irons et al. (2011) found no differences between VGPs and nVGPs at either low or high perceptual loads. Relatedly, in an additional singleton task, VGPs and nVGPs showed no differences in singleton filtering (Cain \& Mitroff, 2011). To explore another aspect of distractor filtering, here we examined trial history in a low-load flanker task (Eriksen \& Schultz, 1979; Kerns et al., 2004).

The final ability we tested was logical memory (i.e., memory for narrative details). We hypothesized that, while logical memory is important for daily life, it would not be altered by action video game experience. Thus, this test was intended as a control for possible global differences (e,g., in motivation) that might exist between groups (e.g., Fleck \& Mitroff, 2008). Differential performance in this task would argue for the presence of one or more global group differences, while no difference would suggest that the effects seen in the other two tasks are specific to the cognitive processes employed by those tasks.

\section{Experiment 1: Task switching and flanker task}

Method

Participants A total of 44 members of the University of California, Berkeley, community participated for cash payment or partial course requirement fulfillment. Participants were divided into groups based on their responses to a questionnaire that assessed expertise (1-7) and experience (h/week) with six different types of video games. The VGP group included those who played at least $6 \mathrm{~h} /$ week of games, primarily first-person shooting (FPS) or action games, and ranked themselves at least a 5 in FPS or action video game expertise. The $\mathrm{nVGP}$ group included those who played less than $2 \mathrm{~h} /$ week of FPS and action games and ranked themselves a 2 or lower on FPS and action game expertise. The VGP group contained 23 participants (mean age $=20.8$ years, all males), and the nVGP group contained 21 participants (mean age $=22.5$ years, 8 males, 13 females). All participants also completed Experiment 2, as well as three other tasks, to be reported elsewhere (Landau, Cain, \& Shimamura, 2012).

Stimuli and procedure Stimuli were presented on Dell Dimension 8100 computers with 18 -in. CRT monitors using EPrime Software. Participants were seated approximately 18 in. away from the monitor. Each stimulus array consisted of three arrows arranged horizontally at the center of a black screen, each pointing either left or right (Fig. 1) subtending approximately $3.0^{\circ} \times 1.4^{\circ}$ of visual angle, with arrows separated by $0.4^{\circ}$. The task for each trial was indicated by the arrows' color: Participants were to report via keypress the direction indicated by the center arrow for blue stimuli (pro-response trials) or the opposite direction for yellow stimuli (anti-response trials) using the same keys for both

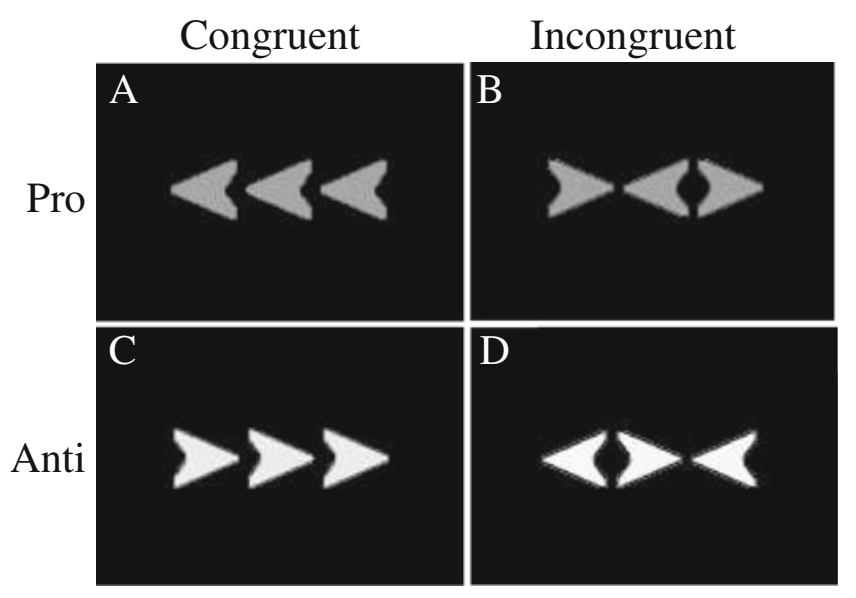

Fig. 1 Stimuli for Experiment 1. Participants were instructed to respond to the direction indicated by the center arrow (pro-response) when the arrows were blue ( $\mathrm{A}$ and $\mathrm{B}$, shown as dark gray) and to indicate the direction opposite the one indicated by the center arrow (anti-response) when the arrows were yellow (C and D, shown as light gray), but always to ignore the outside, flanking arrows. There were a total of four stimulus types: (a) pro-response congruent, (b) proresponse incongruent, (c) anti-response congruent, ans (d) antiresponse incongruent. The correct response to each of the stimuli pictured here would be a left keypress. Participants also saw an equal number of mirror images of these stimuli, indicating right keypresses 
tasks ("z" and "")). On half of the trials, the outside (i.e., flanking) arrows pointed in the same direction as the central target arrow (congruent trials), and on the other half of trials the outside arrows pointed in the opposite direction (incongruent trials). Participants were instructed to ignore the outside arrows and to respond only to the central arrow. Each stimulus array was presented for $1,300 \mathrm{~ms}$, followed by a black screen with a central fixation point $\left(1.0^{\circ}\right.$ white filled circle) for 1,800-2,200 ms. Four 85-trial experimental blocks followed an eight-trial practice block.

To assess task history effects, trials were classified on the basis of the tasks of both the current and the previous trial; for example, for a pro-response switched trial, a proresponse was required on the current trial, whereas an antiresponse had been required on the previous trial. Equal numbers of pro-response repeated, pro-response switched, anti-response repeated, and anti-response switched trials were presented. Similarly, to examine sequential flanker effects, trials were reclassified on the basis of the congruencies of the current and the previous trial. Equal numbers of congruent repeated, congruent switched, incongruent repeated, and incongruent switched trials were presented.

\section{Results and discussion}

Task switching The results are summarized in Fig. 2a and are detailed in Table 1. Trials with RTs $<200 \mathrm{~ms}$ or $>1,200 \mathrm{~ms}(2.5 \%$ of the total $)$ were excluded from all analyses, and incorrect trials $(4.7 \%$ of the total) were excluded from the RT analysis. RTs were submitted to a $2 \times$ $2 \times 2$ mixed-model ANOVA, with Task Type (pro-response or anti-response) and Task History (repeated or switched) as within-subjects factors, and Group (VGP or nVGP) as a between-subjects factor. We found a significant main effect of task type $\left[F(1,42)=5.65, M S E=781.00, p=.022, \eta_{\mathrm{p}}{ }^{2}=\right.$ .118 , with pro-response trials being faster than antiresponse trials (657 vs. $668 \mathrm{~ms}$ ), and a significant main effect of task history $[F(1,42)=93.98, M S E=477.09$, $\left.p<.001, \eta_{\mathrm{p}}^{2}=.672\right]$, with repeated-task trials being faster than switched-task trials (647 vs. $678 \mathrm{~ms})$. There was no main effect of group: VGPs were not faster than nVGPs overall $\left[F(1,42)=1.41, M S E=32,767.27, p=.242, \eta_{\mathrm{p}}{ }^{2}=\right.$ $.032]$. Task type and task history interacted significantly $[F$ $\left.(1,42)=56.36, M S E=243.33, p<.001, \eta_{\mathrm{p}}{ }^{2}=.596\right]$, with larger switching costs for pro-response than for antiresponse trials (50 vs. $12 \mathrm{~ms}$ ). There was no significant Task History $\times$ Group interaction, suggesting no overall difference in task-switching costs between groups. Importantly, we did find a significant three-way interaction between task type, task history, and group $[F(1,42)=8.96, M S E=243.33 p=$ $\left..005, \eta_{\mathrm{p}}{ }^{2}=.182\right]$. nVGPs had a large switching cost for proresponse trials and a small switching cost for anti-response trials (63 vs. $9 \mathrm{~ms}$ ), while VGPs had a more symmetric pattern (37 vs. $16 \mathrm{~ms})$.

Errors were submitted to the same ANOVA as RTs. There was a significant main effect of task history $[F(1,42)=34.76$, $\left.M S E<0.01, p<.001, \eta_{\mathrm{p}}{ }^{2}=.453\right]$, with pro-response trials being more accurate than anti-response trials $(2.6 \%$ vs. $5.3 \%$ errors). We also found a significant interaction between task type and task history $[F(1,42)=8.49, M S E<0.01, p=.006$, $\left.\eta_{\mathrm{p}}{ }^{2}=.171\right]$, with larger switching costs for pro-response than for anti-response trials (3.5\% vs. $1.9 \%$ errors), consistent with the RT results. No other main effects or interactions were significant (all $p \mathrm{~s}>.1$ ). Notably, no evidence emerged of group differences in speed-accuracy trade-offs, because both groups produced more errors on switched-task trials than on repeated-task trials (VGP switching cost $=2.8 \%$, nVGP switching cost $=2.5 \%$ ).

A potential confounding factor was the uneven gender distribution of the two groups. Gender has long been a concern in video game research, as more males than females tend to play action-oriented games (e.g., Lucas \& Sherry, 2004). The resulting difficulty in recruiting female VGPs has led to many studies recruiting only male participants for cross-sectional analyses. However, both males and females have been included in action video game training studies that have not reported any gender differences after training (e.g., Boot et al., 2008; Green \& Bavelier, 2003; Green, Pouget, \& Bavelier, 2010). This lack of posttraining differences aligns with previous work arguing that gender differences in spatial cognition are likely due to differences in computer experience (Terlecki \& Newcombe, 2005). Furthermore, although the present groups had uneven gender ratios, there was no a priori reason to predict that males and females would differ on task switching. ${ }^{1}$ Thus, the differences observed here are most likely due to uneven video game experience rather than to inherent gender differences.

Flanker task We reanalyzed the same trials organized by flanker congruency (Fig. $2 \mathrm{~b}$ and Table 1). The RTs for correct trials were submitted to a $2 \times 2 \times 2$ mixed-model ANOVA with Flanker Congruency (congruent or incongruent) and Congruency History (repeated or switched) as withinsubjects factors, and Group (VGP or nVGP) as a betweensubjects factor. We found a main effect of flanker congruency $\left[F(1,42)=69.70, M S E=910.16, p<.001, \eta_{\mathrm{p}}{ }^{2}=.635\right]$, with congruent trials being faster than incongruent trials (644 vs.

\footnotetext{
${ }^{1}$ For example, we reanalyzed the results of a previous study using the same paradigm (Cain, 2009, Exp. 2) with participants divided by gender (14 females, 22 males, video game experience unknown). In that study, there were no differences in the switching costs between males and females for either pro-response $[t(34)=0.23, p=.822]$ or anti-response $[t(34)=0.78, p=.438]$ trials.
} 
A

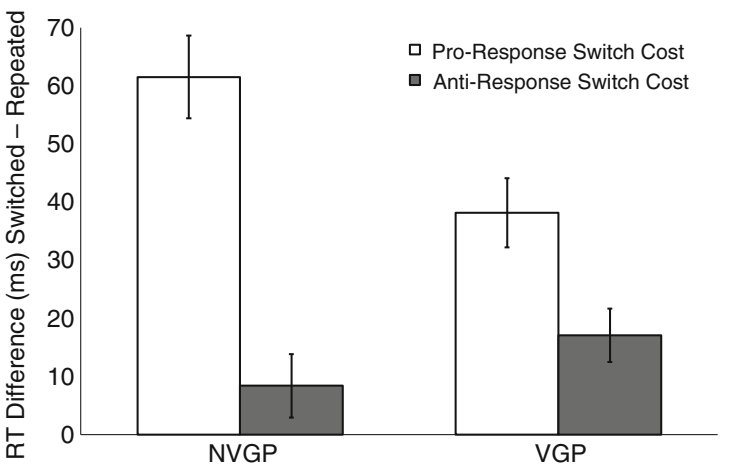

B

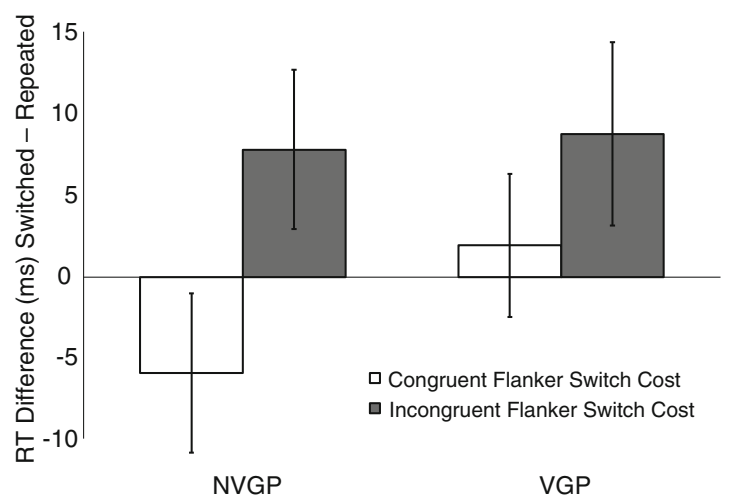

Fig. 2 Response time results for Experiment 1, broken down by (a) task switching and (b) flanker congruency. Error bars represent the standard errors of the means

$685 \mathrm{~ms})$, and an interaction between flanker congruency and congruency history, with a larger flanker effect for trials following a congruent trial than for trials following an incongruent trial (45 vs. $37 \mathrm{~ms}$ ). No other main effects or interactions were significant, indicating no overall or trial history differences between VGPs and nVGPs.

\section{Experiment 2: Logical memory}

One consideration in cross-sectional studies is nonspecific differences: One group may be overall more intelligent, faster, or more motivated than the other, independent of the question of interest. Participant recruitment bias is of particular concern for video game studies. Many of the present participants were contacted using prescreening data and were blind to the purpose of the study, ${ }^{2}$ while others were recruited via advertisements that specifically asked for VGPs and nVGPs. A concern about the latter method is that VGPs may be motivated to perform especially well and that nVGPs may not have such motivation (Boot et al., 2011).

To look for such effects, we assessed logical memory (i.e., memory for the details of a story), which has previously been used to demonstrate performance differences due to stress (e.g., VonDras, Powless, Olson, Wheeler, \& Snudden, 2005) and medical malingering (e.g., Langeluddecke \& Lucas, 2003). We predicted that logical memory would not be enhanced by action video game experience, thus revealing any motivational differences between the groups.

\footnotetext{
${ }^{2}$ Even this method was not without potential bias: During debriefing, one participant reported that he had guessed that he was recruited for his video game experience, as he felt his other prescreening answers were unremarkable.
}

Method

Participants All participants in Experiment 1 participated in Experiment 2. Two VGPs' scores were unanalyzable, due to a procedural error. Thus, the analysis included 21 VGPs and 21 nVGPs.

Stimuli and procedure Participants listened to two auditorily presented 30-s stories, and after each story were immediately asked to verbally recall as many details as possible. Recall was unspeeded, and no cues or prompts were provided. After recall for the second story, that story was replayed, and participants were again asked to recall. During each recall, an experimenter checked off the remembered details on a scoring sheet.

\section{Results and discussion}

The total scores across all three recollections (possible range $=$ 0-72) were summed and converted to scale scores (possible range $=1-19)$ to remove any age-based effects (Wechsler, 1997). The overall sample contained a great deal of variability in memory ability (raw range $=25-62$, scaled range $=4-15$ ), but these differences were not associated with video game playing. nVGPs performed slightly, but nonsignificantly, better than VGPs $[\mathrm{nVGP}$ mean $=10.62, \mathrm{VGP}$ mean $=9.86$; $t(40)=1.04, p=.307]$. The scaling procedure did not mask group differences, since none appeared in the raw-score differences, either $[t(40)=0.97, p=.334]$.

As predicted, logical memory performance was no better in VGPs than in nVGPs. By itself, this says little about VGPs' performance. However, given that this lack of difference was in an effortful task performed in the same session as Experiment 1, the task-switching performance differences are less likely to be due to general intelligence, recruitment effects, or motivation. 


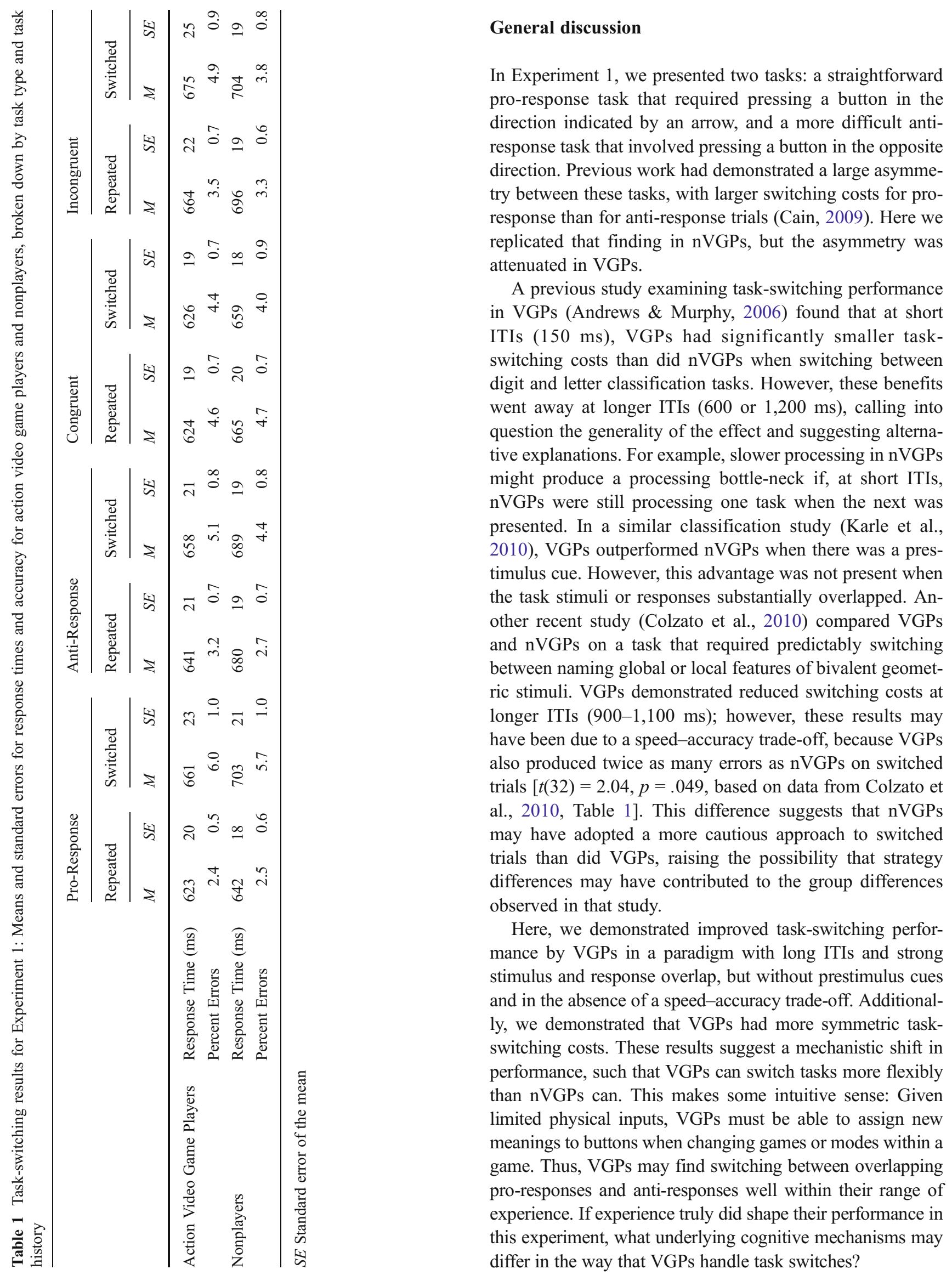


According to the positive-priming account of taskswitching costs (Yeung \& Monsell, 2003; Yeung, Nystrom, Aronson, \& Cohen, 2006), when a task needs to be performed, the representation of the task is activated and remains active until a different task needs to be performed. When a second task is activated, the first task must be inhibited to prevent inappropriate responses. This added inhibition step accounts for switching costs in general and for asymmetric costs in particular: A novel task, such as an anti-response task, will carry over more strongly — and will need to be inhibited more strongly - than will a familiar task such as a pro-response, leading to longer switching costs on pro-response switched trials than on anti-response switched trials.

Given this account, VGPs might show more symmetric task switch costs than nVGPs for several reasons. One possibility is that VGPs may activate each task representation less strongly, leading to reduced overall switching costs and reduced task asymmetries, but also to an overall slowdown. We found no direct evidence of overall slowing, but since VGPs typically perform faster than nVGPs (Dye et al., 2009b), the lack of group differences in RTs suggests that VGPs may have responded more slowly than they could have under other circumstances. Alternatively, VGPs may actively try to disengage from the current task set after each trial. Given the balanced numbers of switch and repeat trials, this strategy would pay off half the time and would reduce task switch costs, but at the expense of reducing repetition benefits, producing a pattern similar to that in Fig. 2 .

In Experiment 1, we also examined distractor filtering. Consistent with previous singleton distractor (Cain \& Mitroff, 2011) and low-perceptual-load flanker (Green \& Bavelier, 2003; Irons et al., 2011) results, no differences in filtering abilities were seen between VGPs and nVGPs, even when examining sequential flanker congruency effects. Taken together, these results strongly argue that action video game experience does not improve visual information filtering. The logical memory task in Experiment 2 also revealed no differences between VGPs and nVGPs. Notably, the auditory presentation and verbal responses were dissimilar from the conditions of action video games, and if VGPs were motivated to perform well only in game-like contexts, Experiment 2 might not have revealed motivation differences (Boot et al., 2011). However, considered alongside the lack of a VGP advantage in the flanker effect, this suggests that such global effects as intelligence and motivation cannot account for the task-switching findings and promotes the idea that such differences are specific to the executive control system and are expertise-driven.

Author note This study was supported by NIH Grant DA14110 and NSF Grant BCS-0745835 to A.P.S. Thanks to Bona Kang, Nola Klemfuss, Samuel Sakhai, Sadaf Sareshwala, Bailey Seymore, and Katharina Volkening for assistance with data collection.

\section{References}

Allport, A., Styles, E., \& Hsieh, S. (1994). Shifting intentional set: Exploring the dynamic control of tasks. In C. Umiltà \& M. Moscovitch (Eds.), Attention and performance XV: Conscious and nonconscious information processing (pp. 421-452). Cambridge, MA: MIT Press, Bradford Books.

Andrews, G., \& Murphy, K. (2006). Does video game playing improve executive functioning? In M. A. Vanchevsky (Ed.), Frontiers in cognitive psychology (pp. 145-161). New York, NY: Nova Science.

Boot, W. R., Blakely, D. P., \& Simons, D. J. (2011). Do action video games improve perception and cognition? Frontiers in Cognition, 2, 226. doi:10.3389/fpsyg.2011.00226

Boot, W. R., Kramer, A. F., Simons, D. J., Fabiani, M., \& Gratton, G. (2008). The effects of video game playing on attention, memory, and executive control. Acta Psychologica, 129, 387-398. doi:10.1016/j.actpsy.2008.09.005

Cain, M. S. (2009). Asymmetric rule switching effects and cognitive conflict: A behavioral, fMRI, and training investigation (Doctoral dissertation). University of California, Berkeley, CA.

Cain, M. S., \& Mitroff, S. R. (2011). Distractor filtering in media multitaskers. Perception, 40, 1183-1192. doi:10.1068/p7017

Chisholm, J. D., Hickey, C., Theeuwes, J., \& Kingstone, A. (2010). Reduced attentional capture in action video game players. Attention, Perception, \& Psychophysics, 72, 667-671. doi:10.3758/ APP.72.3.667

Clark, K., Fleck, M. S., \& Mitroff, S. R. (2011). Enhanced change detection performance reveals improved strategy use in avid action video game players. Acta Psychologica, 136, 67-72. doi:10.1016/j.actpsy.2010.10.003

Colzato, L. S., van Leeuwen, P. J. A., van den Wildenberg, W. P. M., \& Hommel, B. (2010). DOOM'd to switch: Superior cognitive flexibility in players of first person shooter games. Frontiers in Cognition, 1, 8. doi:10.3389/fpsyg.2010.00008

Donohue, S. E., Woldorff, M. G., \& Mitroff, S. R. (2010). Video game players show more precise multisensory temporal processing abilities. Attention, Perception, \& Psychophysics, 72, 1120-1129. doi:10.3758/APP.72.4.1120

Dye, M. W. G., Green, C. S., \& Bavelier, D. (2009a). The development of attention skills in action video game players. Neuropsychologia, 47, 1780-1789. doi:10.1016/j.neuropsychologia.2009.02.002

Dye, M. W. G., Green, C. S., \& Bavelier, D. (2009b). Increasing speed of processing with action video games. Current Directions in Psychological Science, 18, 321-326. doi:10.1111/j.14678721.2009.01660.x

Eriksen, C. W., \& Schultz, D. W. (1979). Information processing in visual search: A continuous flow conception and experimental results. Perception \& Psychophysics, 25, 249-263. doi:10.3758/ BF03198804

Fleck, M. S., \& Mitroff, S. R. (2008). Videogamers excel at finding rare targets [Abstract]. Journal of Vision, 8(6), 313a. doi:10.1167/ 8.6.313

Green, C. S., \& Bavelier, D. (2003). Action video game modifies visual selective attention. Nature, 423, 534-537. doi:10.1038/ nature 01647

Green, C. S., Pouget, A., \& Bavelier, D. (2010). Improved probabilistic inference as a general learning mechanism with action video games. Current Biology, 20, 1573-1579. doi:10.1016/j. cub.2010.07.040

Irons, J. L., Remington, R. W., \& McLean, J. P. (2011). Not so fast: Rethinking the effects of action video games on attentional capacity. Australian Journal of Psychology, 63, 224-231. doi:10.1111/j.1742-9536.2011.00001.x

Jersild, A. T. (1927). Mental set and shift. New York, NY: Archives of Psychology. 
Karle, J. W., Watter, S., \& Shedden, J. M. (2010). Task switching in video game players: Benefits of selective attention but not resistance to proactive interference. Acta Psychologica, 134, 70-78. doi:10.1016/j.actpsy.2009.12.007

Kerns, J. G., Cohen, J. D., MacDonald, A. W., III, Cho, R. Y., Stenger, V. A., \& Carter, C. S. (2004). Anterior cingulate conflict monitoring and adjustments in control. Science, 303, 1023-1026. doi:10.1126/science. 1089910

Landau, A. N., Cain, M. S., \& Shimamura, A. P. (2012). Voluntary and involuntary attention in video game players. Manuscript in preparation.

Langeluddecke, P. M., \& Lucas, S. K. (2003). Quantitative measures of memory malingering on the Wechsler Memory Scale-Third edition in mild head injury litigants. Archives of Clinical Neuropsychology, 18, 181-197. doi:10.1016/S0887-6177(01)00195-0

Lucas, K., \& Sherry, J. L. (2004). Sex differences in video game play: A communication-based explanation. Communication Research, 31, 499-523. doi:10.1177/0093650204267930

Monsell, S. (2003). Task switching. Trends in Cognitive Sciences, 7 , 134-140. doi:10.1016/S1364-6613(03)00028-7

Monsell, S., Yeung, N., \& Azuma, R. (2000). Reconfiguration of taskset: Is it easier to switch to the weaker task? Psychological Research, 63, 250-264. doi:10.1007/s004269900005

Murphy, K., \& Spencer, A. (2009). Playing video games does not make for better visual attention skills. Journal of Articles in Support of the Null Hypothesis, 6, 2.

Okagaki, L., \& Frensch, P. A. (1994). Effects of video game playing on measures of spatial performance: Gender effects in late adolescence. Journal of Applied Developmental Psychology, 15, 33-58.

Spector, A., \& Biederman, I. (1976). Mental set and mental shift revisited. American Journal of Psychology, 89, 669-679.

Terlecki, M. S., \& Newcombe, N. S. (2005). How important is the digital divide? The relation of computer and videogame usage to gender differences in mental rotation ability. Sex Roles, 53, 433-441.

Tsoi, L., Koopman, S., \& Wilmer, J. B. (2011). Video-game training improves smooth pursuit precision. Paper presented at the meeting of the Vision Sciences Society, Naples, FL.

VonDras, D. D., Powless, M. R., Olson, A. K., Wheeler, D., \& Snudden, A. L. (2005). Differential effects of everyday stress on the episodic memory test performances of young, mid-life, and older adults. Aging and Mental Health, 9, 60-70. doi:10.1080/ 13607860412331323782

Wechsler, D. (1997). WMS-III: Wechsler memory scale (3rd ed.). San Antonio, TX: Psychological Corp.

Wylie, G., \& Allport, A. (2000). Task switching and the measurement of "switch costs." Psychological Research, 63, 212-233. doi: $10.1007 / \mathrm{s} 004269900003$

Yeung, N., \& Monsell, S. (2003). Switching between tasks of unequal familiarity: The role of stimulus-attribute and response-set selection. Journal of Experimental Psychology: Human Perception and Performance, 29, 455-469. doi:10.1037/0096-1523.29.2.455

Yeung, N., Nystrom, L. E., Aronson, J. A., \& Cohen, J. D. (2006). Between-task competition and cognitive control in task switching. Journal of Neuroscience, 26, 1429-1438. doi:10.1523/JNEURO SCI.3109-05.2006 